\title{
Evaluación del nivel de estrés en leoncillos (Cebuella pygmaea) mediante la medición de cortisol en heces
}

\author{
Estefanía Espinosa Mata $^{1}$, Stella de la Torre ${ }^{1 *}$, Venancio Arahana ${ }^{1}$, María de Lourdes Torres $^{1}$ \\ ${ }^{1}$ Universidad San Francisco de Quito. Colegio de Ciencias Biológicas y Ambientales. Diego de Robles s/n \\ y Vía Interoceánica, Quito - Ecuador. \\ *Autor principal/Corresponding author,e-mail: sdelatorre@usfq.edu.ec \\ Editado por/Edited by: Diego F. Cisneros-Heredia, Ph.D.(c) \\ Recibido/Received: 2015/10/05. Aceptado/Accepted: 2015/11/01. \\ Publicado en línea/Published on Web: 2015/12/30. Impreso/Printed: 2015/12/30.
}

Evaluation of the stress level in pygmy marmosets (Cebuella pygmaea) by measurement of fecal cortisol

\begin{abstract}
The pygmy marmoset (Cebuella pygmaea) is the smallest platyrrhine primate. It lives in gallery forests in the Upper Amazon basin of Colombia, Ecuador, Peru, Bolivia and Brazil. Although all the Ecuadorian primate species are threatened by human activities, the pygmy marmoset is one of the most vulnerable due to its high specialization in diet and habitat. It is included in the list of vulnerable species in Ecuador. Anthropogenic alteration of the habitats is a stress factor for pygmy marmosets. In this context this study aimed to establish a protocol to measure cortisol concentration in feces (ng/g), as an indicator of the stress level of the individuals, and to make a preliminary comparison of the levels of cortisol and stress of three populations of pygmy marmosets in Amazonian Ecuador. Fifty two fecal samples from the wild populations of Tiputini and San Pablo, and one captive population from Puyo were analyzed. The samples analyzed had a dry weight between 0.05 and $0.07 \mathrm{~g}$. The biological validation made with the fecal samples of an individual quarantined, showed that cortisol levels were higher in times closer to its death. The preliminary results suggest that the captive population from Puyo has higher levels of stress than the wild populations. These results have to be confirmed by analyzing a larger number of samples with information about the time of fecal deposition, the sex, age and previous activities of the individuals.
\end{abstract}

Keywords. Cebuella pygmaea, stress, cortisol in feces, primates, Ecuador.

\section{Resumen}

El leoncillo (Cebuella pygmaea), es la especie más pequeña de primate platirrino. Habita en bosques de la Amazonía alta en Colombia, Ecuador, Perú, Bolivia y Brasil. Aunque todas las especies de primates en el Ecuador están amenazadas por las actividades humanas, ésta es una de las más vulnerables, debido a la especificidad de su alimentación y hábitat. C. pygmaea actualmente ha sido incluida en la lista de especies vulnerables en el Ecuador. La alteración antropogénica de los hábitats es un factor de estrés para los leoncillos. En este contexto, los objetivos de esta investigación fueron establecer un protocolo para medir el cortisol en heces, como un indicador del nivel de estrés de los individuos, y realizar una comparación preliminar de los niveles de cortisol y estrés de tres poblaciones de leoncillos en la Amazonía ecuatoriana. Se analizaron 52 muestras provenientes de las poblaciones silvestres de Tiputini y San Pablo y de una población en cautiverio en Puyo. Las muestras analizadas tuvieron pesos secos entre 0.05 y $0.07 \mathrm{~g}$. La validación biológica realizada con muestras de heces de un individuo en cuarentena, evidenció que los niveles de cortisol fueron más elevados en los días más próximos a su muerte. Los resultados preliminares sugieren que la población en cautiverio de Puyo tiene niveles de estrés significativamente más altos que los de las poblaciones silvestres. Estos resultados deben ser confirmados analizando un mayor número de muestras de las que se tenga información completa sobre la hora de deposición y sobre los individuos de los que se tomaron las muestras.

Palabras Clave. Cebuella pygmaea, estrés, cortisol en heces, primates, Ecuador.

\section{Introducción}

Cebuella pygmaea, conocido también como leoncillo o tití pigmeo, pertenece a la familia Cebidae y a la subfa- milia Callitrichinae [1]. Es el primate más pequeño de América del Sur [2], habita en bosques siempre verdes de tierras bajas inundables de la Amazonía alta en Colombia, Ecuador, Perú, Bolivia y Brasil [3]. Los leon- 


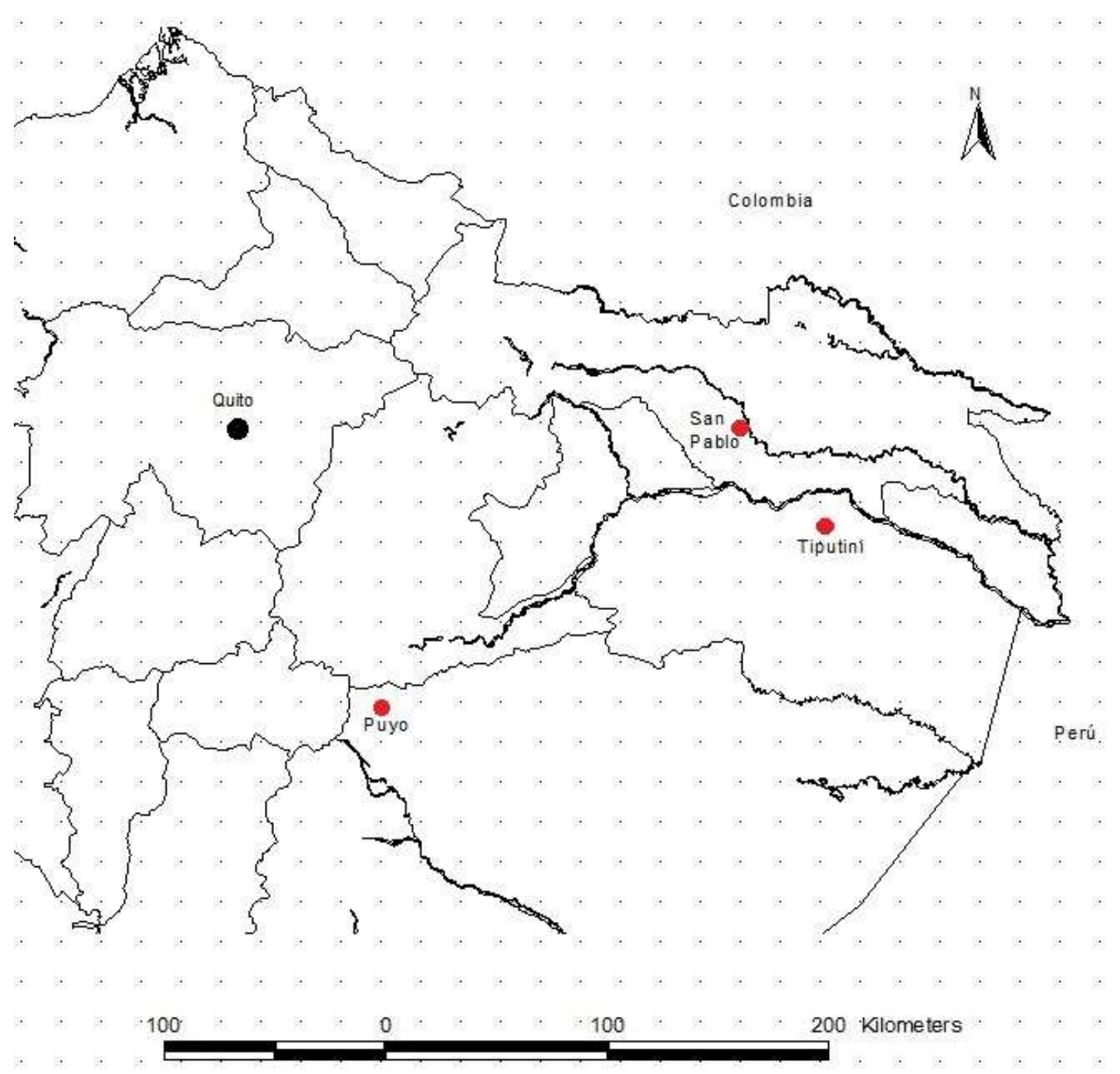

Figura 1: Ubicación de las tres poblaciones de Cebuella pygmaea en la Amazonía ecuatoriana (San Pablo, Puyo y Tiputini (4 grupos - T1, T2, T3 y T5).

cillos son importantes para mantener la estructura y la dinámica de los ecosistemas por su papel en las redes tróficas como presas y depredadores [3].

Aunque todas las especies de primates silvestres en el Ecuador están amenazadas por las actividades humanas, C. pygmaea es una de las más vulnerables, debido a la especificidad de su alimentación y hábitat $[3,4,5]$. Por esta razón en el 2011 fue incluida en la lista de especies vulnerables de mamíferos del Ecuador [6]. Esta especie se encuentra también incluida en el Apéndice II de la Convención sobre el Comercio Internacional de Especies Amenazadas (CITES) lo que indica que su comercio internacional debe ser controlado cuidadosamente [7].

Los primates son especialmente sensibles a los efectos de la fragmentación del hábitat. El $90 \%$ de sus poblaciones vive en bosques tropicales [7], de manera que la destrucción y fragmentación de este ecosistema ha sido una de las causas principales del declive mundial de este grupo de mamíferos [8]. La fragmentación del bosque tiene múltiples consecuencias para los primates; además de la restricción espacial a sus actividades, la reducción de la disponibilidad de alimentos, la alteración de la demografía y la dinámica de la población, aumenta la susceptibilidad a enfermedades, desequilibrando la homeostasis y causando estrés en los individuos [9]. El estrés se define como un estado de alteración de la homeostasis de un individuo debido a factores ambientales, como las temperaturas extremas, escasez de agua y/o alimento, o factores fisiológicos como el estado reproductivo [9]. Además puede ser desencadenado por factores psico-sociales como peleas, subordinación social o carencia de control sobre una situación dada [10]. La presencia continua de los seres humanos, la provisión artificial de los alimentos y las perturbaciones ambientales también son causa de estrés [9]. La acción de estos estímulos produce cambios en la actividad del sistema nervioso autónomo y el eje hipotálamo-pituitariaadrenocortical-HPA [11].

La respuesta al estrés es fisiológicamente costosa y pue- 


\begin{tabular}{cccc}
\hline & Muestras & g muestra seca & Concentración ng cortisol / g muestra \\
\hline 1 & PY1.3 & 0,05 & 799,44 \\
2 & PY1.2 & 0,05 & 738,61 \\
3 & PY1.1 & 0,1 & 365,64 \\
\hline
\end{tabular}

Tabla 1: Validación biológica del protocolo de extracción de cortisol usando un individuo (PY1) cuyas características eran conocidas. Las muestras fueron rotuladas de acuerdo a la fecha de colección de la muestra, siendo PY1.1 la muestra más lejana a la muerte del individuo y PY1.3 la más cercana $($ rho $=1)$.

de tener efectos negativos sobre los procesos esenciales del organismo cuando se da durante períodos prolongados. El estrés puede afectar el éxito reproductivo de los individuos $\mathrm{y}$, como consecuencia a la dinámica y viabilidad de una población [9]. Por tanto, desde el punto de vista de conservación de la vida silvestre, y en este caso de los leoncillos, es importante evaluar el nivel de estrés al que están sometidos estos animales, con el objetivo de encontrar sus causas junto con las medidas tendientes a reducirlo.

En la mayoría de los mamíferos, incluidos los primates, el principal glucocorticoide es el cortisol, el cual se incrementa en situaciones de estrés, por lo que constituye un marcador deseable cuando se busca determinar niveles de estrés [12]. Hay que tener en cuenta que existen variaciones individuales relacionadas con el sexo, condiciones reproductivas o condición social, que pueden afectar las concentraciones de cortisol [13]. El método tradicional de medición del cortisol es en el plasma sanguíneo, pero al ser éste un método invasivo, en la actualidad se prefiere determinar cortisol en otros productos corporales como saliva, orina y heces. Los métodos no invasivos son deseables ya que los animales no necesitan ser capturados y se pueden obtener varias muestras sin alterar el comportamiento o estado endócrino del individuo [14].

En este contexto, los objetivos de esta investigación fueron: 1) establecer un protocolo para medir el cortisol en heces de leoncillos, como un indicador del nivel de estrés de los individuos y; 2) realizar una comparación preliminar de los niveles de estrés de leoncillos de tres poblaciones en la Amazonía ecuatoriana. Dos de estas son poblaciones en estado silvestre que habitan en bosques con diferente grado de fragmentación; la tercera es una población en cautiverio.

\section{Metodología}

\section{Áreas de Estudio}

Las áreas donde se colectaron las muestras de heces de leoncillos fueron: (1) Puyo, Centro de Rescate Yanacocha, Provincia de Pastaza (coordenadas UTM, zona 18. Datum WGS 84, 9838712 N - 167707 E), (2) San Pablo, Provincia de Sucumbíos (9969781 N - 341056 E) y (3) Estación de Biodiversidad Tiputini, Provincia de Orellana (9929379 N - 371669 E) (Fig 1).

En Puyo, en el Centro de Rescate Yanacocha, estudiamos un grupo en cautiverio de 7 individuos en el 2013 y 12 individuos en el 2014. En San Pablo estudiamos un grupo silvestre de 6 individuos en el 2011, esta área ha sufrido en los últimos años un alto grado de fragmentación de los bosques [3, 4]. Finalmente en Tiputini, estudiamos 4 grupos silvestres: T1 (con 3 individuos en el 2012 y 5 individuos en el 2013), T2 (con 6 individuos en el 2012), T3 (con 6 individuos en el 2013) y T5 (con 5 individuos en el 2014), esta población no está expuesta a los efectos de la fragmentación ni a actividades de cacería.

\section{Métodos}

Las heces en todos los grupos fueron colectadas en mallas colocadas bajo los principales árboles de comida de los grupos. Los grupos fueron observados a lo largo de todo el día para cubrir todo el período de actividad de los leoncillos, en períodos de 3 a 6 horas de observación continua. Las heces eran recolectadas en cuanto se detectaba su presencia en la red. Se trató siempre de identificar a los individuos que las producían pero esto solo fue posible en pocas ocasiones. En todas las muestras se registró la fecha y el grupo al que pertenecían, en algunas muestras se registró la hora de deposición. Las muestras fueron guardadas en alcohol etílico al $90 \%$ en condiciones de sombra hasta su transporte al Laboratorio de Biotecnología de la USFQ.

La extracción de cortisol a partir de heces se realizó siguiendo el protocolo propuesto por Ziegler y colaboradores [15]. Para realizar este proceso se secó la muestra a $28{ }^{\circ} \mathrm{C}$ por 6 horas, luego se la pulverizó y se añadió agua y etanol (1:1), se mezcló con el vórtex y se centrifugó por 25 minutos a $13200 \mathrm{rpm}$, colectándose el sobrenadante. Se preparó una curva estándar de 8 puntos (0-0.04-0.1-0.2-0.4-1.0-2.0-10.0 ng/ml) a partir de cortisol puro, provisto en el kit "Cortisol EIA Kit" de Oxford Biomedical. Para reducir sesgos en las mediciones de cortisol solo se analizaron las muestras con pesos secos entre $0.05 \mathrm{~g}$ y $0.07 \mathrm{~g}$ (en pruebas preliminares detectamos concentraciones consistentemente altas de cortisol en muestras menores a $0.05 \mathrm{~g}$ ). Mediante ensayos por inmunoabsorción ligada a enzimas (ELISA), se determinaron, por duplicado, las concentraciones de cortisol de cada una de las muestras siguiendo el protocolo del kit. Las lecturas del microplato se realizaron usando un fotocolorímetro DYNEX MRX y los valores fueron transformados a ng de cortisol/g de peso seco de la muestra.

\section{Análisis de datos}

Para evaluar la validez biológica del protocolo de cuantificación de los niveles de cortisol, se analizaron las concentraciones de cortisol de tres muestras de heces 
de un individuo en cuarentena de la población de Puyo (PY1), colectadas en fechas diferentes durante un periodo de dos meses previo a su deceso. La muestra PY1.1 fue colectada a fines de mayo 2013, la muestra PY1.2 a principios de junio 2013, y la muestra PY1.3, que fue la muestra más próxima a la muerte del individuo, a mediados de junio 2013. Se realizó una correlación de Spearman entre las concentraciones de cortisol y el orden cronológico en el que las muestras fueron tomadas (1: fecha más distante del deceso, 3: fecha más próxima al deceso).

Con las concentraciones de cortisol de 52 muestras con pesos secos entre 0.05 y $0.07 \mathrm{~g}$ de las tres poblaciones, se realizó un análisis de varianza (ANOVA) y una prueba de separación de medias de Tukey; el software utilizado para estos análisis fue R. Para evaluar, de forma preliminar, diferencias entre horas de deposición y sexo de los animales, se compararon los promedios de cortisol de aquellas muestras en las que se tenía información sobre el período del día en el que fueron depositadas (06h00 a $12 \mathrm{~h} 00$ vs. 12h01 a 18h00) y entre muestras de las cuales se conocía el sexo del individuo (hembras vs. machos).

\section{Resultados}

\section{Validación biológica}

De las tres muestras analizadas del individuo del Puyo en cuarentena, la muestra PY1.3 tuvo la mayor concentración de cortisol con 799,44 ng/g muestra seca (Tabla 1). El coeficiente de correlación de Spearman entre la concentración de cortisol y la fecha de colección de las muestras evidencia una correlación perfecta $(\mathrm{rho}=1)$, aunque no significativa debido al bajo número de mues$\operatorname{tras}(n=3)$.

\section{Diferencias interpoblacionales en la concentración de cortisol}

Los niveles de cortisol de las muestras de la población en cautiverio del Puyo fueron significativamente más altos que los de las muestras de las poblaciones silvestres de San Pablo y Tiputini $\left(\mathrm{F}_{2,49}=9.46, \mathrm{p}=0.00033\right.$, Tukey test Puyo vs. Tiputini $\mathrm{p}=0.0043848$, Puyo vs. San Pablo $\mathrm{p}=0.0006135$ ). Estos resultados sugieren que el cautiverio tiene un efecto significativo sobre los niveles de cortisol (y de estrés) en esta especie de primate. En el test de separación de medias de Tukey no se encontraron diferencias significativas entre San Pablo y Tiputini (Fig 2).

\section{Diferencias por hora de deposición de la muestra y sexo en concentraciones de cortisol}

El número de muestras analizadas para evaluar los efectos de la hora de deposición de la muestra y el sexo del individuo sobre las concentraciones de cortisol fue bajo (n $06 \mathrm{~h} 00$ a $12 \mathrm{~h} 00=5, \mathrm{n} 12 \mathrm{~h} 01$ a $18 \mathrm{~h} 00=2$; n hembras $=4, \mathrm{n}$ machos $=3$ ) por lo que no realizamos pruebas

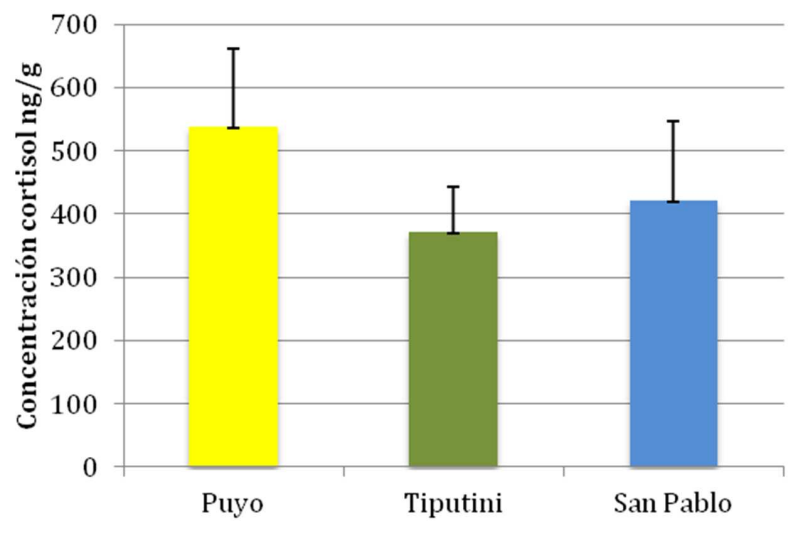

Figura 2: Promedios ( \pm desviaciones estándar) de las concentraciones de cortisol en heces de las tres problaciones de $C$. pygmaea en estudio (n Puyo = 18 n, San Pablo = 11, n Tiputini = 23).

estadísticas con estos datos. Se registraron niveles de cortisol más altos en heces producidas y colectadas en horas de la tarde (12h01 a 18h00) (Fig 3) y en heces de hembras (Fig 4).

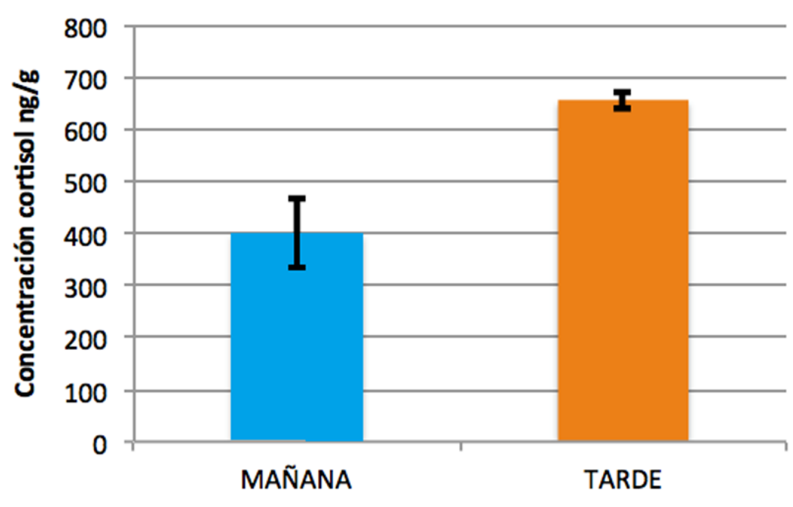

Figura 3: Concentración de cortisol en muestras de heces de individuos de $C$. pygmaea según la hora de deposición (n mañana: $06 h 00$ a $12 \mathrm{~h} 00=5$, n tarde: $12 \mathrm{~h} 00$ a $18 \mathrm{~h} 00=2$ ).

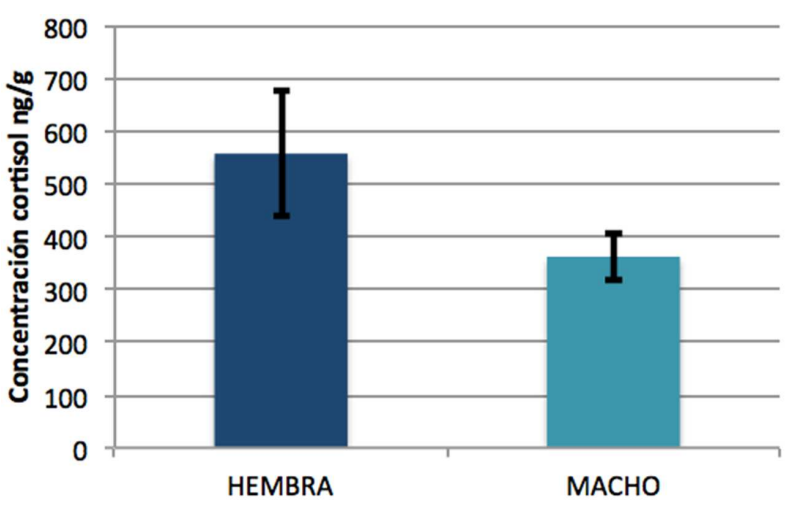

Figura 4: Concentración de cortisol en muestras de heces de individuos de $C$. pygmaea según el sexo $(n$ hembras $=4, n$ machos $=$ 3).

\section{Discusión}

Cebuella pygmaea es una de las especies de primates más amenazadas en el Ecuador debido a su alta especialización de dieta y de hábitat. Las actividades antropogénicas como la construcción de carreteras, viviendas, 
cultivos y tala de árboles han reducido severamente su hábitat [3]. Este impacto humano puede tener repercusiones en el comportamiento, el éxito reproductivo y la viabilidad de las poblaciones y puede verse reflejado en los niveles de estrés que estos primates experimentan [3].

La comparación preliminar realizada entre las tres poblaciones sugiere que el cautiverio es un factor de estrés importante para esta especie. Varias investigaciones demuestran que animales silvestres que son sometidos a cautiverio suelen tener niveles de corticosterona más altos en comparación con los individuos silvestres de la misma especie $[16,17,18]$. Sin embargo, en estos análisis es importante también considerar el tiempo de aclimatación y las condiciones ambientales del cautiverio [19]. Por otro lado, el hecho de que los niveles de cortisol no hayan sido significativamente diferentes entre las poblaciones de San Pablo (bosques altamente fragmentados) y Tiputini (bosques sin fragmentación antropogénica) sugiere que existen factores naturales que generan estrés en los leoncillos aun en bosques con mínimo impacto humano. Sin embargo, es también posible que estos resultados sean consecuencia del bajo número de muestras analizadas que no permitió controlar las variaciones individuales de los niveles de cortisol.

En este contexto, es importante continuar monitoreando los niveles de cortisol tanto de grupos de leoncillos en cautiverio, como en estado silvestre. Al relacionar las concentraciones de cortisol con factores del ambiente físico y social (demográfico) podremos identificar los principales factores de estrés para mejorar las condiciones de vida de los animales en cautiverio y evaluar el impacto de factores ambientales antropogénicos, como la fragmentación de los bosques, y naturales, como la depredación o la competencia interespecífica, en poblaciones silvestres de esta especie.

Estudios realizados con distintas especies de primates, y en otros animales, demuestran que son varios factores los que pueden alterar las concentraciones de cortisol. Un estudio realizado con muestras de sangre, indica que en especies diurnas, los niveles de cortisol disminuyen gradualmente durante todo el día para reducirse al mínimo justo antes de que el animal descanse por la noche. Los altos niveles de cortisol al despertar probablemente sirven para movilizar las reservas de energía en preparación para la actividad diaria [20, 21]. Sin embargo, un estudio mostró que para los titíes comunes (Callithrix jacchus), los niveles más altos de cortisol en heces ocurrían en la tarde, lo cual concuerda con nuestro estudio. Aunque esto podría parecer inesperado ya que este animal es una especie diurna, es posible atribuir este resultado al tiempo que el cortisol se mantiene en los productos fecales en el intestino antes de ser excretado fuera del cuerpo [21, 22]. Así los niveles de cortisol en heces indican en realidad las concentraciones de cortisol circulante que se produjeron en el cuerpo del individuo algunas horas antes [21]. Esto podría explicar las mayores concentraciones de cortisol registradas en nuestro estudio en muestras producidas en la tarde. El bajo número de muestras en el que se basa nuestro análisis no permite llegar a resultados concluyentes. Nuevos análisis son necesarios para confirmar o refutar este posible patrón y sus causas. Así mismo, en estudios realizados con $C$. jacchus se ha determinado que las hembras tienen valores de referencia de cortisol tres veces mayores que los machos, independientemente de si éstas se están reproduciendo o no [23]. Aunque nuestros resultados coinciden con los de este estudio, necesitamos analizar un mayor número de muestras para conocer más sobre las diferencias entre sexos en los niveles de cortisol y estrés en esta especie de primate.

La validación biológica del protocolo de extracción y cuantificación de cortisol en heces del leoncillo, permitió evaluar como se relaciona la condición física del animal con la concentración de cortisol de las muestras de un individuo en cuarentena. Nuestros resultados sugieren que los desajustes fisiológicos que llevaron a la muerte del individuo fueron un factor de estrés que actuó en forma aditiva. Estos resultados evidencian también la efectividad de los análisis para detectar cambios en los niveles de cortisol de acuerdo a las condiciones fisiológicas de los individuos.

\section{Agradecimientos}

El estudio fue realizado gracias a la colaboración de Charles T. Snowdon del Departamento de Psicología de la Universidad de Wisconsin, Toni Ziegler del Wisconsin Regional Primate Center, al Laboratorio de Biotecnología Vegetal y del Laboratorio de Enfermedades Tropicales de la Universidad San Francisco de Quito.

La investigación de campo se realizó bajo los permisos 0010-2012-FAU-MAE-DPO-PNY, 006-2013-IC-FAUDPAP/MAE, 012-2014-FAU-MAE-DPAO-PNY y 004IC-FAU-DPS/NA del Ministerio del Ambiente a Stella de la Torre. Agradecemos el apoyo en la investigación de campo de Hernán Payaguaje, Alfredo Payaguaje, Anna del Valle, Anna Zango, Robert Burton, Gary Judas, Marcela Garzón y Lucía Valles, así como de los directores y personal de la Estación de Biodiversidad Tiputini y del Centro de Rescate Yanacocha.

\section{Referencias}

[1] Tagliaro C.; Cruz, M.; Schneider, H.; Stanhope, I.; Stanhope, M. 2000. "Molecular studies of Callithrix pygmaea (Primates, Platyrrhini) based on transferrin intronic and ND1 regions: implications for taxonomy and conservation". Genetics and molecular biology, 23(4): 729-737.

[2] Bairrao, E. 2010. "Cebuella pygmaea. Guía de manejo para Callitrícidos”. (Asociación Europea de Zoológicos y Acuarios) EAZA, ZooParc de Beauval. Segunda Edición. 
[3] de la Torre S.; Yépez, P.; Snowdon, C. 2009. "Conservation Status of Pygmy marmosets (Cebuella pygmaea) in Ecuador". Chapter 22. En: "The Smallest Anthropoids: The Marmoset/Callimico Radiation”. L.C. Davis , S.M. Ford, L. Porter (eds.). Springer Science-Business Media: 451-464.

[4] de la Torre S.; Yépez, P.; Snowdon, C.; Payaguaje, A. 2007. "Ecología, comportamiento y conservación del leoncillo Callithrix (Cebuella) pygmaea en Tierras Secoya". En: Caminando en el sendero, hacia la conservación del ambiente y la cultura Secoya. S. de la Torre y P. Yépez (eds). Fundación VIHOMA. Quito: 91-98.

[5] Yépez, P.; de la Torre, S.; Snowdon, C. 2005. "Interpopulation differences in exudate feeding of Pygmy marmosets in Ecuadorian Amazonia". American Journal of Primatology, 66: 145-158.

[6] Tirira, D. G. 2011. "Libro Rojo de los mamíferos del Ecuador". $2^{a}$ edición. Publicación especial sobre los mamíferos del Ecuador 8. Fundación Mamíferos y Conservación. Pontificia Universidad Católica del Ecuador \& Ministerio del Ambiente del Ecuador, Quito.

[7] de la Torre, S.; Rylands, A. 2008. "Cebuella pygmaea. The IUCN Red List of Threatened Species". Version 2014.2. http://www.iucnredlist.org/details/ $41535 / 0$.

[8] Vegas-Carrillo, S. 2008. "Efectos de la transformación del hábitat en la conducta y niveles de estrés de Alouatta palliata mexicana". Tesis doctoral. Universidad de Barcelona: Barcelona.

[9] Rangel-Negrín A.; Alfaro, J.; Valdez, R.; Romano, M.; Serio-Silva, J. 2009. "Stress in Yucatan spider monkeys: effects of environmental conditions on fecal cortisol levels in wild and captive populations". Animal Conservation ZSL: 496-502.

[10] Álvarez, C.; Arias, L. 2005. "Validación y aplicación de la prueba ELISA para medir cortisol fecal". Medicina Veterinaria. COLCIENCIAS: Colombia, 10: 53-64.

[11] Romero, M.; Uribe, L.; Sánchez, J. 2011. "Stress biomarkers as indicators of animal welfare in cattle beef farming". BioSalud, 10(1): 71-87.

[12] Brousset, D.; Galindo, F.; Valdez, R.; Romano, M., Schuneman, A. 2005. "Cortisol in saliva, urine and feces: non-invasive assessment of wild animals". Etiología, fauna Silvestre y animales de laboratorio, Universidad Nacional Autónoma de México: México D.F.: 335:337.

[13] Keay, J.; Jatinder, B.; Matthew, C.; Gaunt, B.; Taranjit, K. 2006. "Fecal glucocorticoids and their metabolites as indicators of stress in various mammalian species: a literature review". Journal of Zoo and Wildlife Medicine, 37(3): 234-244.

[14] Goymann, W.; Mostl, E.; Van't Hof, T.; East, M.; Hofer, H. 1999. "Noninvasive fecal monitoring of glucocorticoids in spotted hyenas, Crocuta crocuta". General and Comparative Endocrinology, 114: 340-348.
[15] Ziegler, T.; Scheffler, G.; Snowdon, C. 1995. "The relationship of cortisol levels to social environment and reproductive functioning in female cotton top tamarins, Saguinus oedipus". Hormones and Behavior, 29: 407424.

[16] Marra, P. P.; Lampe, K. T.; Tedford, B. L. 1995. "Plasma corticosterone levels in two species of Zonotrichia sparrows under captive and free-living conditions". Wilson Bulletin, 107: 296-305.

[17] Romero, L.; Wingfield, M. 1999. "Alterations in hypothalamic- pituitary-adrenal function associated with captivity in Gambelõs white-crowned sparrows (Zonotrichia leucophrys gambelii)". Comparative Biochemistry and Physiology B, 122: 13-20.

[18] Washburn, B. E.; Millspaugh, J. J.; Schulz, J. H.; Jones, S. B.; Mong, T. W. 2003. "Using fecal glucocorticoids for stress assessment in mourning doves". Condor, 105: 696-706.

[19] Wingfield, J. C.; Smith, J. P.; Farner, D. S. 1982. "Endocrine responses of white-crowned sparrows to environmental stress". Condor, 84: 399-409.

[20] Coe C. L.; Levine, S. 1995. "Diurnal and annual variation of adrenocortical activity in the squirrel monkey". American Journal of Primatology, 35: 283-292.

[21] Cross, N.; Rogers, L. 2004. "Diurnal cycle in salivary cortisol levels in Common Marmosets". Centre for neuroscience and animal behaviour, School of Biological Biomedical and Molecular Science. University of New England: 134-139.

[22] Sousa, M. B. C.; Ziegler, T. E. 1998. "Diurnal variation on the excretion patterns of fecal steroids in common marmoset (Callithrix jacchus) females". American Journal of Primatology, 46: 105-117.

[23] Ferreira, J.; Cordeiro, M.; Sousa, M.; Freire, M. 2010. "Morning and afternoon patterns of fecal cortisol excretion among reproductive and non-reproductive male and female common marmosets, Callithrix jacchus". Biological Rhythm Research, 32(2): 159-167. 